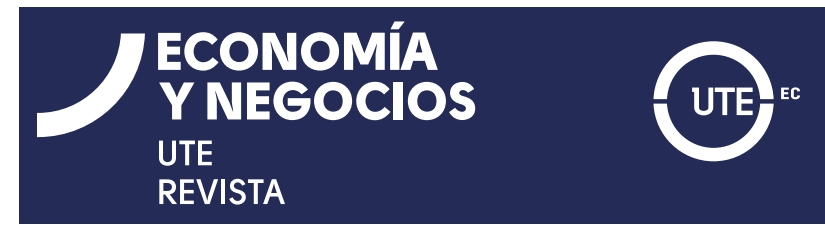

Economía y Negocios, 2021, 12(02), 52-63.

http://revistas.ute.edu.ec/index.php/economia-y-negocios

\section{ARTÍCULO DE INVESTIGACIÓN}

ISSN-e: 2602-8050

Recibido (Received): 2021/07/26 Aceptado (Accepted): 2021/10/20

Publicado (Published): 2021/12/06

https://doi.org/10.29019/eyn.v12i2.957

\title{
La Innovación como Herramienta de Gestión Comercial en las Pymes de Santo Domingo de los Tsáchilas
}

\section{The Innovation as a Commercial Management Tool in SMEs in Santo Domingo de los Tsáchilas}

\author{
Renato Patricio ERAZO-RIVERA ${ }^{1}$ (D), José Armando PANCORBO-SANDOVAL' (iD, \\ Sonia Emilia LEYVA-RICARDO ${ }^{2}$ (iD) y Ángela Elena BARBA-MOSQUERA' ${ }^{1}$ (iD)
}

1. Universidad UTE, Facultad de Ciencias Administrativas. Santo Domingo, Ecuador.

2. Universidad UTE, Facultad de Ingeniería e Industrias. Santo Domingo, Ecuador.

\section{Resumen}

El actual escenario disruptivo en la economía ecuatoriana incentiva a las pymes a generar innovaciones en sus modelos de gestión que les permitan obtener ventajas competitivas. Por medio de este estudio se pretende conocer, a partir de un análisis sistémico, el grado de adopción de la innovación y de las herramientas de las TIC en las pymes de la ciudad de Santo Domingo (Ecuador).

Para el desarrollo del estudio se utilizó el enfoque metodológico mixto, cualitativo y cuantitativo. Mediante la aplicación de encuestas y de la revisión documental se puede valorar cómo los empresarios de esta ciudad utilizan de las TIC.

Los resultados del estudio muestran un escaso uso de la tecnología existente para el desarrollo de estrategias gerenciales innovadoras, consecuencia del bajo nivel de cultura empresarial y de la aversión al riesgo de los empresarios, resultado ya reportado en estudios precedentes.

Palabras clave

Estrategia empresarial, innovación, gestión comercial, investigación documental.

\section{Abstract}

The current disruptive scenario in the Ecuadorian economy encourages SMEs to generate innovations in their management models that allow them to obtain competitive advantages. Through this study, it is intended to know, based on a systemic analysis, the degree of adoption of innovation and ICT tools in SMEs in the city of Santo Domingo (Ecuador).

For the development of the study, the mixed, qualitative and quantitative methodological approach was used. Through the application of surveys and documentary review, it is possible to assess how entrepreneurs in this city use ICT.

The results of the study show little use of existing technology for the development of innovative management strategies, a consequence of the low level of business culture and the risk aversion of entrepreneurs, a result already reported in previous studies.

\section{Keywords}

Business strategy, innovation, commercial management, documentary research.

Códigos de clasificación JEL: L10, M10, M30, O10 


\section{Introducción}

Las pymes se convierten en una pieza central que debe garantizar la viabilidad y eficacia de la transformación a un sistema empresarial innovador centrado en el uso de las nuevas tecnologías, al generar una nueva dinámica de desarrollo que permita un crecimiento económico continuo e innovador, además de tecnológico, incluyente y sostenible (Estrada et al., 2019).

El Reporte de Economía y Desarrollo del Banco de Desarrollo de América Latina (Corporación Andina de Fomento [CAF], 2018) muestra que los bajos índices de productividad en Ecuador y en América Latina, en general, se deben principalmente a factores relacionados con: el capital humano, el grado de competencia entre las empresas, el acceso a los insumos, la cooperación entre las firmas, las relaciones laborales, el financiamiento, la brecha de género, un mal emparejamiento entre las habilidades de los trabajadores y sus tareas, y una gran concentración de la fuerza de trabajo en empleos informales y de baja productividad. Asimismo, muchas empresas se encuentran al margen del proceso de modernización y las microempresas, en su gran mayoría, son ineficientes, entre otras razones, por la escasa utilización de las TIC en el proceso productivo.

Indudablemente las innovaciones de las pymes en su desarrollo deben considerar los preceptos del Manual de Oslo (Organisation for Economic Co-operation and Development [OECD] \& Eurostat, 2017), en el cual se definen cuatro tipos de innovaciones: en el producto, en el proceso, en el marketing y en la organización. Estos preceptos se aplican tanto a la industria como a los servicios, incluidos los que prestan instituciones públicas. En el manual se considera a la innovación como un proceso en red en el que las interacciones entre los agentes generan nuevos conocimientos y tecnología. Asimis- mo, se plantea que los vínculos habituales entre empresa, proveedores y clientes se amplían en los procesos de innovación a otras relaciones, tales como: centros de investigación, enseñanza superior y entidades públicas y privadas de desarrollo.

Jácome y King (2013) señalan que las pymes constituyen el sector más productivo en la economía de un país, por el impacto que tienen en nivel de empleo y de aporte a la productividad tanto en países desarrollados como en los países en vías de desarrollo. Esto se refleja en el Producto Interno Bruto (PIB), con una tasa del más del $25 \%$; además de que las pymes son generadoras de empleo, debido a que abarcan un $70 \%$ de la Población Económicamente Activa (PEA) del país. En Ecuador, las microempresas generan el $39 \%$ de los empleos, el $17 \%$ corresponde a las pequeñas y $14 \%$, a las medianas (Ron Amores \& Sacoto Castillo, 2017, p.12).

La escasa utilización de estrategias de innovación en las pymes de Latinoamérica ocasiona que se dificulte el posicionamiento de productos y procesos y que haga respondiendo a una ventaja competitiva. A su vez, se vuelve evidente la poca importancia que se les concede a los procesos de I+D y a la generación y aplicación de patentes (Rivas, 2019; Dini \& Stumpo, 2018). En Ecuador existen tres grandes obstáculos para la innovación:

a. Falta de apoyo fiscal permanente por medio de políticas públicas;

b. Falta de inversión en la capacidad de habilidades de largo plazo, y

c. Falta de resultados creativos, lo cual deriva en una insuficiencia de patentes.

En el país, según la clasificación que establece el Instituto Nacional de Estadísticas y Censo, (INEC), la adquisición de maquinaria y equipo y la capacitación constituyen las actividades de innovación en las que más se interesan las empresas ecuatorianas, con 
el $31.22 \%$ y $20.18 \%$ respectivamente, y en un menor porcentaje en investigación y desarrollo, software, hardware, asistencia técnica, todas en un promedio del 13 \% (INEC, 2018).

El territorio objeto de estudio es Santo Domingo, una ciudad ecuatoriana que forma parte de la tercera provincia del país (Santo Domingo de los Tsáchilas), tanto por el número de habitantes como por ser un gran centro económico favorecido por su ubicación geográfica en el país. Este territorio está ligado históricamente a las actividades agropecuarias, las cuales representaron el $10.32 \%$ de las ventas totales de la ciudad en el año 2018, y al comercio, tanto al por menor como al por mayor, el cual representaron el $61.61 \%$ de las ventas totales de la ciudad en 2018. Respecto a la actividad agrícola, son múltiples los cultivos que se producen, entre los que se destacan aquellos que están vinculados con la exportación, tales como: banano y plátano, $37.7 \%$ de las ventas agrícolas totales; cacao, $2.8 \%$; palma africana, $36.5 \%$, y piña $17.83 \%$ (Sistema de Información Local [SIL], 2020). La mayor extensión de cultivos está dedicada a estos productos; además de otros tales como: palmito, abacá, yuca, pimienta y maracuyá. El sector ganadero también es importante porque aquí está ubicada la segunda feria ganadera más importante del país, en la que se comercializan en cada sesión de feria 3000 cabezas de ganado bovino, 2000 de ganado porcino y 300 equinos (SIL, 2020). Por último, están las actividades agropecuarias que ocupan el 20.3 \% de la Población Económicamente Activa (PEA) de Santo Domingo (SIL, 2020).

En cuanto al comercio, este genera el $26.4 \%$ de la ocupación en la ciudad. En 2018, se reportaron ventas por el valor de 1385976998 USD (SIL, 2020). El dinamismo de este sector responde a factores geográficos y poblacionales. La provincia de Santo Domingo de los Tsáchilas es paso obligatorio entre las regiones Costa y Sierra, al estar atravesada por la carretera que las comunica, y porque en ella habitan personas de todas las provincias del Ecuador. Los subsectores más importantes en el ámbito comercial son los de vehículos a motor y repuestos; artículos para el hogar, muebles y electrodomésticos; telefonía móvil e informática y negocios de importación de este tipo de artículos. Asimismo, se destaca su rol como centro logístico en la comercialización de pescado y mariscos procedente de las zonas pesqueras (Manabí-Esmeraldas). Por último, tiene un incipiente sector turístico que está compuesto por 275 establecimientos que generan 1451 plazas de empleo (INEC, 2018). De este sector se destacan 1224 establecimientos entre hoteles y restaurantes con una representación amplia de la gastronomía ecuatoriana (INEC, 2018), además de complejos recreacionales ubicados cerca de los ríos (conocidos como balnearios).

En 2018, la ciudad contaba con una PEA de 198247 personas. De esta población, apenas el $40.2 \%$ tenía un empleo formal; un $18.6 \%$ estaba en el subempleo, y un $38.6 \%$ se ubicaba en otras formas de empleo (SIL, 2020). Del total de la PEA, el desempleo no alcanzó el $2.6 \%$. En cuanto a formación, el $31 \%$ de la población total de Santo Domingo (440 909 personas) cursó estudios básicos; el $22 \%$, secundarios, y el $9 \%$, estudios superiores o de posgrado. Hay que destacar que un $23 \%$ ha cursado estudios primarios, además de que existe un $4 \%$ de la población sin instrucción alguna pero que está alfabetizada (SIL, 2020).

Si bien la economía del territorio es dinámica, la innovación y el uso de tecnologías 4.0 no lo caracterizan, pese a la amplia cobertura de las conexiones a internet (tanto fijas como móviles) y al uso extendido de smartphones (Pancorbo Sandoval et al., 2018). Estas herramientas no se usan para generar innovación o mejorar la competitividad por el lado de la promoción o los canales 
de distribución. Además, las estrategias empresariales de los nuevos emprendimientos y de los ya consolidados se basan en copiar modelos exitosos, lo que genera fenómenos como la aparición de un sinnúmero de locales comerciales que exhiben la misma mercadería o se dedican al mismo giro de negocio, pero sin aportar mayor diferenciación que la competencia por el precio. Los autores también han reportado resistencia al cambio y a la introducción de nuevas tecnologías. Un ejemplo es que, según Global Enterpeneurship Monitor (GEM) (ESPAE, 2020), 3 de cada 4 emprendedores ofertan productos que no son nuevos ni siquiera en el ámbito local; además de que el $76.6 \%$ de los emprendimientos oferta procedimientos que localmente tampoco son nuevos. El alcance de los emprendimientos, según el GEM Ecuador (ESPAE, 2020) también es significativo: el $60.9 \%$ de los emprendimientos en zonas urbanas y el $86.9 \%$ en las zonas rurales no tienen alcance nacional ni internacional. Todo ello conlleva a una alta tasa de mortalidad de los emprendimientos (Pancorbo Sandoval et al., 2018; Martínez González-Tablas, 2008) que podría remediarse con la introducción de las TIC.

En 1983, Smith y Miner (Smith \& Miner, 1983) ya establecían una tipología del emprendedor que distingue a los emprendedores artesanos de los oportunistas. Los oportunistas son más adaptativos y orientados al crecimiento del negocio y al manejo del arte de vender y las redes de negocios, además de estar más motivados y tener deseo de logro (Brunswick, 2014).

La situación descrita coincide con lo reportado por otros autores para América Latina. El no uso de tecnología de forma intensiva contribuye al desarrollo del denominado empresario zombi, un fenómeno del escenario latinoamericano. Caicedo-Asprilla (2020), explica que estos empresarios son aquellos que tienen problemas en la estabilidad de sus negocios, especialmente por la ausencia de una estructura tecnológica adecuada. Esta categoría coincide con el empresario artesano descrito por Smith y Miner (Smith \& Miner, 1983; Brunswick, 2014), porque la acción del empresario se inclina hacia la comodidad, a la vez que rehúye de la visión, tenacidad y capacidad emprendedora cuando esta evoluciona a la habilidad de crear y manejar sistemas, y a controlar y crear infraestructura. En consecuencia, este comportamiento obstaculiza llegar a un sistema regional de innovación (SIL, 2020). En contraste, en un estudio de Cordero Linzán (2019), se indica que un mayor fomento del emprendimiento genera más capacidades innovadoras en la región.

En las pymes ecuatorianas se puede hacer referencia al modelo señalado por la denominada teoría de la innovación abierta (Álvarez-Aros \& Álvarez Herrera, 2018), el cual contempla dos formas de actividades dentro de la innovación abierta: la innovación abierta entrante y la innovación abierta saliente. La primera se refiere a la transferencia de tecnología, donde las empresas supervisan su entorno para incorporar la tecnología y los conocimientos en su base de conocimientos internos. La segunda, por el contrario, se refiere a la transferencia de tecnología hacia el exterior, donde las empresas buscan a las organizaciones externas más adecuadas para comercializar una determinada tecnología y que permitan aportar con el uso de tecnologías.

Los autores de este estudio coinciden con los resultados de Carpio-Gallegos y Miralles (2020) en que la adquisición tecnológica influye en la capacidad de innovar productos; sin embargo, si dicha tecnología se emplea poco o de forma inadecuada no se mejorará la competitividad. Esto ocurre en muchas ocasiones por ausencia del nivel cultural y/o técnico requerido para ello, y podría solventarse con formación y capacitación continua. 
Teniendo en cuenta lo anterior, se plantea como objetivo de este estudio conocer, a partir de un análisis sistémico, el grado de adopción de la innovación y de las herramientas de las TIC por parte de las pymes de la ciudad de Santo Domingo de los Tsáchilas, en Ecuador.

\section{Materiales y métodos}

Se partió de la revisión de los siguientes documentos y estudios:

a. Innovación e industria 4.0 en las micro y pequeñas empresas en América Latina: Resultados de investigación en las micro y pequeñas empresas latinoamericanas. Este fue llevado a cabo en 2020, en colaboración con la Red Latinoamericana de Administración y Negocios (RELAYN).

b. Tesis de pregrado Plan estratégico de marketing digital para la gestión comercial de las pymes en el sector comercial de la ciudad de Santo Domingo de los Tsáchilas 2018, Camacho (2019).

c. Información documental primaria de fuentes internacionales y nacionales.

La información formal se centra en los resultados del proyecto ejecutado por Fernández Hernández et al. (2020) en colaboración con la RELAYN, además de la información derivada del mismo proyecto con datos cuantitativos, que constituye la denominada información tabular (Pancorbo Sandoval et al., 2018). También se consideró el estudio de Posada et al. (2018) para el modelo de abordaje de las pymes y su análisis sistémico.

Por medio de esta investigación se pudo diagnosticar el grado en el que las pymes han integrado e implementado acciones de innovación en la región de Santo Domingo de los Tsáchilas, de acuerdo con los cuatro tipos propuestos en el Manual de Oslo (OECD \& Eurostat, 2007). De igual manera, desde la perspectiva del análisis sistémico, se buscó relacionar las características de la empresa con la prevalencia en la adopción de estos principios y acciones.

Los siguientes resultados son parte de la investigación anual de la RELAYN. En esa investigación se estudiaron 103 poblaciones en México, Colombia, Argentina, Perú y Ecuador y se aplicaron 42076 cuestionarios. La muestra permite comparar los resultados obtenidos en el estudio con una población de referencia con características socioculturales similares. Se considera un estudio transversal, pues la recolección de datos se llevó a cabo en un solo momento; además, es descriptivo porque tiene como fin indagar en el nivel de las variables en las pymes de la región objeto de estudio.

El estudio se hizo mediante un cuestionario aplicado a 344 directores de pymes. Se definió como pymes a cualquier organización con fines de lucro que tenga, al menos, entre 2 y 50 personas trabajando en ella; además, se consideró como director a la persona que toma la mayoría de las decisiones en la empresa. El tamaño de la muestra requerido para la población, obtenido con un nivel de confianza del $95 \%$ y un margen de error del $5 \%$, así como asumiendo proporciones esperadas del $50 \%$. En consecuencia, se ha considerado que el número de empresas que participaron en el estudio es de un tamaño inferior al necesario, por lo que los resultados deberán interpretarse con cautela. La información fue recopilada por alumnos de la Universidad UTE, ${ }^{1}$ quienes fueron capacitados tanto en la aplicación del cuestionario como en la captura en la plataforma bajo la supervisión de los autores.

En paralelo a la interpretación de los resultados del estudio de RELAYN, los auto-

1 Los datos fueron recopilados del 16 de junio al 25 de junio de 2020, en la Universidad UTE, sede Santo Domingo. 
res de este estudio consideraron válido analizar información tabular procedente de distintas fuentes técnicas para sustentar con más pertinencia los resultados.

Como complemento al estudio anterior, se consideraron los principales resultados de la tesis de pregrado de Camacho (2019) en colaboración con los autores de este artículo científico como parte del grupo científico GEPDIT (Grupo de Estudios en Planificación y Desarrollo Inteligente de Territorios) en el periodo 2017-2019. En el caso de la tesis del ingeniero Camacho, se consideraron los resultados de la aplicación de un sondeo a 100 empresarios miembros de la Cámara de Comercio de Santo Domingo en cuanto a la utilización de las herramientas del marketing digital para el desarrollo de su gestión comercial. Asimismo, creyeron oportuno contrastar la información derivada de diferentes fuentes especializadas, lo cual posibilitó complementar el análisis.

\section{Análisis y resultados}

Con el objetivo de contextualizar el panorama de América Latina y el Caribe en torno a la inversión que hace la región en I+D en relación con otra, se presentan los siguientes cuadros que muestran la relación que existe entre el PIB y la inversión en I+D (ver figura 1).

Figura 1. Relación que existe entre el PIB y la inversión en $1+D$
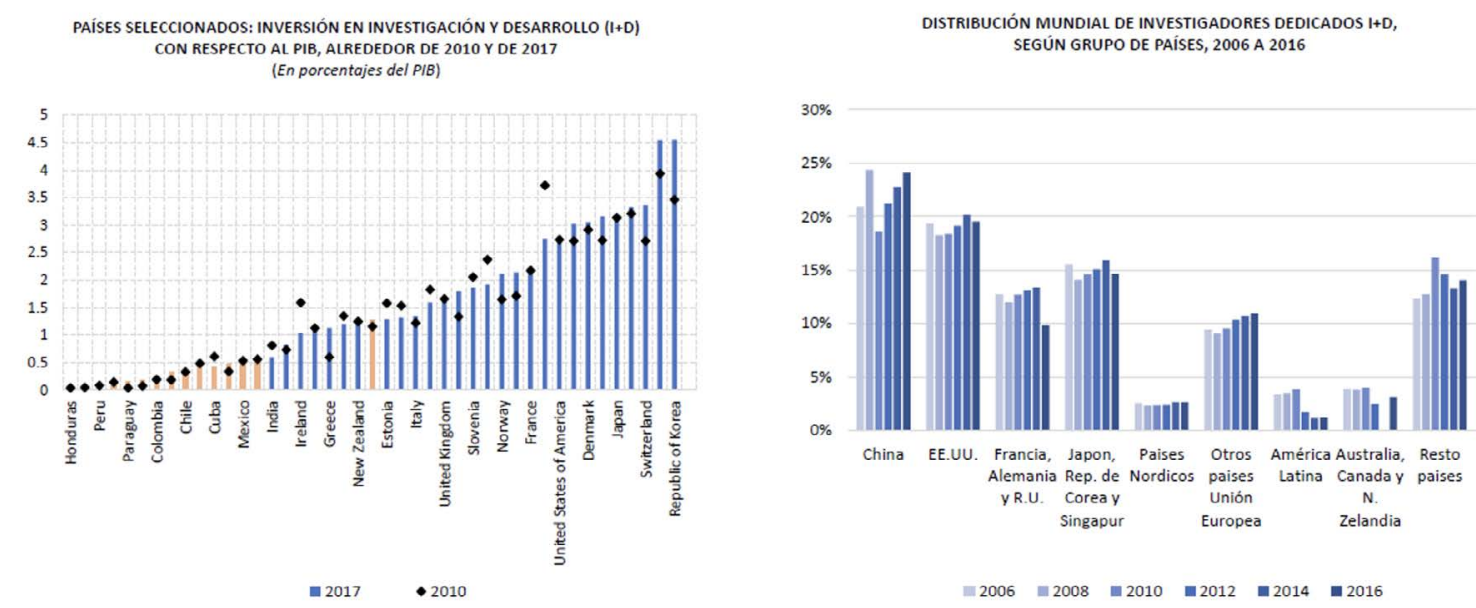

Nota: Tomado de CEPAL (2017): Observatorio COVID-19 en América Latina y el Caribe Impacto económico y social.

Como se mencionó antes, la inversión que hacen los países latinoamericanos en $1+D$ es escasa. Dado que la apuesta por la I+D articula iniciativas tanto en el ámbito público como en el privado, y considerando que el $98.28 \%$ son pymes en la región objeto de estudio, el impacto de la reducción del PIB en I+D perjudicará aún más a las pymes.

El decrecimiento de la tasa de variación del PIB en América Latina fue de - $7.1 \%$ en 2020 (Fondo Monetario Internacional
[FMI], 2021), cifra menor a la estimada inicialmente por la Comisión Económica para América Latina (Naciones Unidad \& CEPAL, 2021) de -9.1\% (FMI, 2021). La reorientación de los esfuerzos económicos de los gobiernos regionales para enfrentar los efectos de la COVID-19 y el parón obligado de la economía por las medidas de confinamiento no constituye un escenario favorable para las pymes del Ecuador, donde se registró un descenso del -9 \% de su PIB, en 2020, lue- 
go de haber crecido solo un $0.1 \%$ en 2019 , según datos de la Naciones Unidas y CEPAL (2021), y en particular para las de Santo Domingo de los Tsáchilas.

La pandemia ha generado unas condiciones macroeconómicas muy difíciles para los actores económicos ecuatorianos, especialmente para las pymes. A continuación, se expondrán las condiciones tecnológicas con las que se enfrentaban al escenario descrito un año después de iniciar la pandemia. Los datos corresponden a los resultados obtenidos por los autores en el trabajo con la RELAYN y por el ingeniero Camacho en su investigación respectivamente. Los datos no difieren demasiado, lo cual confirma el perfil tecnológico de las pymes santodomingueñas.

El perfil tecnológico que tienen los administradores y las pymes en Santo Domingo establece que un $48.4 \%$ tienen páginas web, el $67.4 \%$ usa la banca por internet, un $63.4 \%$ usan programas de computadora para la administración, un $79.4 \%$ usa WhatsApp en el celular, y otras redes sociales el $70.5 \%$. En cuanto al uso de las herramientas de oficina la puntuación más alta fue el uso de buscadores (76.4 \% y $80.2 \%$ ); no obstante, en cuanto comienza a ser más especializada la aplicación de las TIC, los porcentajes de uso de estas se reducen, como en el caso del uso de bases de datos (44.5 y $36.3 \%$ ) o SAP/ERP/MRP (42.2 \% y $33.6 \%$ ) (Pancorbo Sandoval et al., 2018).

En relación con la evolución del comercio electrónico causada por la pandemia, se puede constatar que las ventas digitales en Ecuador han crecido entre el 2019 y 2020 en un $54 \%$, mientras que los que hacen sus primeras compras en línea crece en un $13 \%$ durante el 2020, tal y como se muestra en el estudio sobre uso de internet en Ecuador (Cañete, 2020; Del Alcázar Ponce, 2021), en el cual también se indica que un $49 \%$ de ecuatorianos prefiere comprar vía Whats-
App; mientras que el $44 \%$ lo hace en otras aplicaciones de celular, y un $35 \%$ opta por adquirir bienes en sitios web.

De acuerdo con el indicador de uso de plataformas digitales de la CAF (2018), Ecuador presenta una puntuación baja con respecto al promedio de Latinoamérica, lo que permite corroborar con los datos recabados por los autores sobre el uso mayoritario de WhatsApp y páginas web para comprar en línea, en detrimento de aplicaciones móviles. Estos datos estarían en consonancia con Aguilar Rascón et al. (2020), para quienes el $84 \%$ de las compras se llevan a cabo en webs y WhatsApp.

Asimismo, a partir del reporte de Del Alcázar Ponce (2021), se puede concluir que las pymes ecuatorianas no apuestan por desarrollar apps nativas para el comercio electrónico, sino que confían en empresas transnacionales, de tal forma que desaprovechan la infraestructura tecnológica que existe para innovar en este tipo de servicios. De igual modo, se hace hincapié en el uso erróneo de WhatsApp como una herramienta de comercio electrónico y no como un canal de comunicación. Esta app está pensada para la comunicación en tiempo real, lo cual resulta más óptimo para la preventa, promoción y posventa que para la compraventa, debido a que la app carece de una pasarela de pago y de herramientas de solicitud automática de pedidos que estén conectadas con un sistema de inventario automatizado. Es por estas razones que se corrobora que se da un uso poco adecuado de una tecnología, con las consecuencias negativas que eso conlleva, tales como: falta de transparencia, equívocos en los pedidos, entre otros).

Aparte, los consumidores han tenido también que adaptarse a las condiciones impuestas por los confinamientos preventivos impuestos por el gobierno, y lo han hecho optando por usar las tecnologías. El índice de resiliencia digital del hogar, creado por la CAF, permite me- 
dir el grado en que las familias han logrado ser resilientes gracias a las TIC en cuatro ámbitos: salud, educación, comercio electrónico y finanzas y banca electrónica (ver tabla 1).

Tabla l. El índice de resiliencia digital del hogar

\begin{tabular}{|l|c|}
\hline \multicolumn{1}{|c|}{ País } & Índice \\
\hline Argentina & 33.87 \\
\hline Bolivia & 6.23 \\
\hline Brasil & 40.59 \\
\hline Colombia & 41.14 \\
\hline República Dominicana & 16.38 \\
\hline Ecuador & 11.53 \\
\hline El Salvador & 12.78 \\
\hline Guatemala & 8.70 \\
\hline Honduras & 9.83 \\
\hline México & 25.63 \\
\hline Panamá & 28.63 \\
\hline Paraguay & 16.90 \\
\hline Perú & 23.33 \\
\hline América Latina (promedio ponderado) & 30.70 \\
\hline OCDE (promedio ponderado) & 53.78 \\
\hline
\end{tabular}

Nota: Tomado de CAF (2020).

Se puede corroborar, basándose en los datos, que la resiliencia digital de los hogares latinoamericanos es menor y que se aleja considerablemente de los países de la OCDE. En este índice el Ecuador se ubica por debajo de la media regional (CAF, 2020), lo que indica que la falta de apuesta digital en las pymes también está reflejada en las familias. Sin embargo, después de un año de la pandemia y tomando en cuenta las últimas medidas decretadas por el Ejecutivo en abril de 2021 -que incluyen confinamientos de viernes a domingo por 30 días y teletrabajo obligatorio durante toda la semana para el sector público y privado (Decreto Ejecutivo 1291, 2021)-, los autores consideran que las pymes deben integrar las nuevas tecnologías no solo para mejorar su desempeño empresarial, sino para estar preparadas para las presentes y futuras medidas de prevención que las autoridades ecuatorianas puedan decretar a futuro, de forma que las pymes puedan paliar o evitar los efectos colaterales de dichas medidas.

\section{Discusión y conclusiones}

Retomando los resultados de esta investigación en colaboración con RELAYN (2000), se puede constatar que el trabajo de campo permitió corroborar los datos oficiales (SIL, 2020) al respecto de cuáles son las actividades principales que se desarrollan en la economía santodomingueña, tales como: la agricultura, ganadería, caza y las actividades de servicios conexas; el comercio, al por mayor y menor de diversos bienes; la venta de partes, piezas y accesorios para vehícu- 
los; textiles, y alimentación (Pancorbo Sandoval et al, 2020; Bonilla et al., 2020).

En la tabla 2 se puede observar que existen diferencias en cuatro variables identificadas entre Santo Domingo de los Tsáchilas y los resultados del estudio en general. De lo que se citó antes, se puede señalar que existe mayor innovación en el mercado, bienes o servicios, recursos humanos y sofisticación Mype 4.0 que a nivel del estudio en general.

En cuanto a los resultados derivados de la tesis de pregrado del ingeniero Camacho, se puede verificar los resultados del estudio de RELAYN. Entre los empresarios consultados, un $70 \%$ desconoce las nece- sidades del marketing digital o no sabe aplicarlo en sus negocios, a pesar de contar con las herramientas para crear diversas tácticas de marketing digital como smartphones, computadores, internet, tablets, entre otros. El $83 \%$ de los encuestados no cuenta con una app móvil de su negocio, y solo el 17 \% sí la posee. Esto puede ser debido a que la población en Santo Domingo no está acostumbrada a usar apps móviles para comprar en la zona (Camacho, 2019). Asimismo, el $51 \%$ de los encuestados sí utiliza banca por internet, mientras que el $49 \%$ no lo hace. Estos porcentajes no tan distantes reflejan la utilidad de emplear este método de pago (Camacho, 2019).

Tabla 2. Diferencias en cuatro variables identificadas entre Santo Domingo

\begin{tabular}{|l|c|c|l|c|}
\hline \multicolumn{1}{|c|}{ Variable } & Ítems & $\begin{array}{c}\text { Santo Domingo } \\
\text { de los Tsáchilas }\end{array}$ & \multicolumn{1}{|c|}{ Nacional $^{\mathbf{a}}$} & p. valor $^{\mathbf{b}}$ \\
\hline Innovación al mercado & 13 & $2.20423(2.13714-2.27133)$ & $1.91525(1.9059-1.92461)$ & 0.0000 \\
\hline Innovación en procesos & 12 & $1.93466(1.8561-2.01322)$ & $1.81186(1.80182-1.8219)$ & 0.5131 \\
\hline $\begin{array}{l}\text { Innovación en bienes } \\
\text { o servicios }\end{array}$ & 5 & $2.14493(2.0709-2.21895)$ & $1.92667(1.91764-1.93571)$ & 0.0007 \\
\hline $\begin{array}{l}\text { Innovación en recursos } \\
\text { humanos }\end{array}$ & 5 & $1.77854(1.68199-1.8751)$ & $1.71602(1.70522-1.72682)$ & 0.0000 \\
\hline Sofistificación Mype 4.0 & 15 & $4.11327(4.01707-4.20947)$ & $3.58734(3.57581-3.59888)$ & 0.0000 \\
\hline
\end{tabular}

Nota: a) Se refiere los resultados obtenidos en 103 zonas de México, Colombia, Argentina, Perú y Ecuador y b) prueba t, con ajuste de Bonferroni (Meek et al., 2007).

En el estudio del ingeniero Camacho se demuestra, además, la escasa utilización de la innovación en la gestión comercial en lo referente al comercio electrónico. El 81 \% de los encuestados carece de una página web, por lo que desaprovecha, por ejemplo, la posibilidad de publicitarse a través de Google, estrategia que tampoco practica el $91 \%$ de los encuestados. En contraste, el 19 \% sí dispone de una página web que ayuda a la gestión comercial de la empresa. Otro dato preocupante es que el $63 \%$ de las empresas no ha comprado ni vendido por medio de internet, el $18 \%$ ha hecho compras y ventas por internet, el $13 \%$ ha vendido a través de internet y solo el $6 \%$ ha comprado por el mismo medio. Estos datos demuestran que los microempresarios desconocen estas estrategias digitales.

En cuanto a estudios técnicos precedentes debe destacarse el de la Escuela de Negocios (ESPAE, 2020) porque permite verificar los resultados anteriores. En este estudio se expone que los emprendimientos en Ecuador están concentrados en las ciudades, con escasa presencia de pymes de alcance nacional o internacional; se plantea que 4 de cada 5 emprendedores fueron mo- 
tivados por la escasez de empleo; y se evidencia el limitado uso de las TIC de forma sistemática, innovación e internacionalización son debilidades del emprendimiento en el país. De igual forma, si bien se puede constatar mejoras en el networking y acceso a financiamiento, estos factores siguen siendo evaluados de forma negativa.

Respecto a los datos de las encuestas desarrolladas, se puede señalar que las pymes de Santo Domingo de los Tsáchilas deben mejorar su competitividad en la Mype 4.0, integrar sistemas de información, desarrollar software para mejorar sus procesos y servicios, integrar monitoreo con sensores remotos conectados por internet, así como concentrarse en la capacitación continua de su personal laboral, además de elaborar o actualizar constantemente el análisis de mercado e introducir mejoras continuas en su gestión de ventas y planes de marketing.

Visto desde una perspectiva latinoamericana, Santo Domingo de los Tsáchilas presenta mayor grado de innovación que otras ciudades del estudio de RELAYN en variables de mercado, bienes o servicios, recursos humanos y sofisticación Mype 4 (Pancorbo Sandoval et al, 2018; Heredia, 2020).

Aquí se ha podido hallar que las variables que más influyen en la innovación de las empresas de la ciudad son las de innovación de mercado, lo que incluye recursos humanos, análisis de mercado y la dirección, gestión de ventas y mercadotecnia. En cuanto a la innovación en procesos, esta se ve afectada por el análisis de mercado, la dirección e innovación.

Las TIC son empleadas por los empresarios de Santo Domingo de los Tsáchilas como un canal para la comercialización, pero no para tomar decisiones o ejecutar labores de inteligencia de mercado. Esto sugiere que las pymes santodomingueñas no consideran la importancia del marketing digital, o que las perciben como un gasto más que una inversión, porque no ven los resultados en el retorno sobre la inversión (ROI). Es más, cuando los empresarios ponen en marcha alguna acción de marketing en redes sociales no les dan seguimiento. Esto hace que no se capten leads adecuadamente y que estos no se conviertan en clientes a corto plazo, ni se fidelice a los ya existentes a largo plazo.

En conclusión, las pymes de la región en estudio deberán mejorar su competitividad en la industria 4.0, concentrarse en la capacitación continua de su personal laboral, así como en elaborar o actualizar constantemente sus análisis de mercado e introducir mejoras en su gestión de ventas y en planes de marketing.

\section{Referencias}

Aguilar Rascón, O.C; Peña Ahumada, N.B., \& Posada Velázquez, A. (2020). Innovación e Industria 2.0 en las micro y pequeñas empresas en América Latina. Tomo II. Mc Graw Hill.

Álvarez-Aros, E. L., \& Álvarez Herrera, M. (2018). Estrategias y prácticas de la innovación abierta en el rendimiento empresarial: Una revisión y análisis bibliométrico. Revista Investigación Administrativa, 47(121). https://bit.ly/2ZIP5cy

Bonilla, A., Durán, G., Bayón, M., \& Abad, K. V. (2020). Santo Domingo de los Tsáchilas: El rentismo y sus efectos en las periferias al sur de la ciudad. FLACSO Ecuador.

Brunswick, G. J. (2014). A chronology of the definition of marketing. Journal of Business \& Economics Research, 12(2), 105-114. https://doi.org/10.19030/jber.v12i2.8523

Caicedo-Asprilla, H. (2020). La producción del conocimiento de las regiones competitivas: Una aproximación basada en modelos de variables latentes. Estudios Gerenciales, 36(155), 177-192. http://doi. org/10.18046/j.estger.2020.155.3257

Camacho, D. (2019). Plan estratégico de marketing digital para la gestión comercial de las pymes en el sector comercial de la ciudad de Santo Domingo de los Tsáchilas 
[Tesis de grado, Universidad UTE]. Respositorio Dspace

Cañete, N. (2020, 22 de marzo). Coronavirus: ¿Cómo apoyar desde el sector de fomento a la innovación y las pymes? Puntos sobre la l: Ciencia, Tecnología e Innovación. https://bit.ly/3m4jeps

Carpio-Gallegos, J.F.D., \& Miralles, F. (2020). Análisis de la innovación tecnológica en empresas manufactureras peruanas de baja y media-baja tecnología. Contaduría y Administración, 64(4), e131. https://bit. ly/31RzrXD

Comisión Económica para América Latina y el Caribe (CEPAL). (2017, 23 de marzo). Es necesario repensar el rol de las mipymes de la región para integrarlas a la cuarta revolución industrial, coincidieron expertos. Naciones Unidas. https://bit.ly/30YMRAF

Cordero Linzán, M.G. (2019). El comercio electrónico e-commerce, análisis actual desde la perspectiva del consumidor en la ciudad de Guayaquil, provincia del Guayas y estrategias efectivas para su desarrollo. [Tesis de Maestría, Universidad Católica de Guayaquil]. Repositorio Universidad Católica de Santiago de Guayaquil. https:// bit.ly/3kmu8Wp

Corporación Andina de Fomento (CAF). (2020). El estado de la digitalización de América Latina frente a la pandemia del COVID-19. CAF. https://bit.ly/3jmekTa

Decreto Ejecutivo 1291. (2021). https://bit. ly/3vHOhMU

Del Alcázar Ponce, J.P. (2021, 1 de agosto). Estado digital de Ecuador: Julio 2021. Mentinno Consultores. https://bit.ly/3mHEO3r

Dini, M., \& Stumpo, G. (2018). Mipymes en América Latina: Un frágil desempeño y nuevos desafios para las políticas de fomento. Comisión Económica para América Latina y el Caribe. Documentos de proyectos (LC/ TS.2018/75/Rev.1) https://bit.ly/3bEHng8

ESPAE Escuela de Negocios. (2020). Global Entrepreneurship Monitor: Ecuador 2019-2020. ESPAE Escuela de Negocios. https://bit. ly/3DUVSsy

Estrada, S., Cano, K., \& Aguirre, J. (2019). ¿Cómo se gestiona la tecnología en las pymes?
Diferencias y similitudes entre micro, pequeñas y medianas empresas. Contaduría y Administración, 64(1), 1-21. https://doi. org/10.22201/fca.24488410e.2019.1812

Fernández Hernández, A., Reyes, Velázquez, A., Demesa Udave, J. C., \& Gómez Vicario, M. Á. (2020). En O. C. Aguilar Rascón, N. B. Peña Ahumada, R. Posada Velázquez (Eds.), Innovación e industria 4.0 en las micro y pequeñas empresas en América Latina: Resultados de investigación en las micro y pequeña empresas latinoamericanas (Tomo II., pp 293-300). McGraw Hill.

Fondo Monetario Internacional (FMI). (2021, 10 de abril). Perspectivas de la economía mundial. Fondo Monetario Internacional. https://bit.ly/2ZfDM5P

Heredia, A. (2020). Políticas de fomento para la incorporación de las tecnologías digitales en las micro, pequeñas y medianas empresas de América Latina: Revisión de experiencias y oportunidades. Documentos de Proyectos (LC/TS.2019/96). Santiago de Chile: Naciones Unidas. https://bit. ly/3b0LF1a

Instituto Nacional de Estadística y Censos (INEC). (2018). Tecnologías de la información y comunicación: Encuesta multipropósitoTIC 2018. https://bit.ly/3B4wXkz

Jácome, H., \& King, K. (Coords.). (2013). Estudios industriales de la micro, pequeña y mediana empresa. Ministerio de Industrias y Productividad; FLACSO Ecuador.

Martínez González-Tablas, Á. (2008). El análisis estructural y sus relaciones con el análisis sistémico y los análisis parciales. $R e$ vista de Economía Mundial, 18, 393-404. https://bit.ly/3b4i1rz

Meek, G. E., Ozgur, C., \& Dunning, K. (2007). Comparison of the $t v s$. Wilcoxon Signed-Rank test for likert scale data and small samples. Journal of Modern Applied Statistical Methods, 6(1), 91-106. https://doi. org/10.22237/jmasm/1177992540

Naciones Unidas \& Comisión Económica para América Latina y el Caribe (CEPAL). (2021). Balance Preliminar de las Economías de América Latina y el Caribe 2020. https:// bit.ly/3jZlddr 
Organisation for Economic Co-operation and Development (OECD) \& Eurostat. (2007). Oslo Manual: Guía para la recogida e interpretación de datos sobre innovación (3. ${ }^{a}$ Ed.). Organisation for Economic Cooperation and Development. https://doi. org/10.1787/9789264065659-es

Pancorbo Sandoval, J., Erazo Rivera, R., Lapeña Sanz, R., \& Leyva Ricardo, S. (2018). Potencial tecnológico de las micro y pequeñas empresas latinoamericanas a partir del análisis sistémico: Caso Santo Domingo, Ecuador. En S. A. Delgado (Coord.), Potencial tecnológico de las micro y pequeñas empresas latinoamericanas a partir del análisis sistémico: Tomo II (pp. 587-601). Fontamara.

Posada, R., Aguilar, O. C., \& Peña, N. B. (2018). Método. En S.A. Delgado (Coord.), Potencial tecnológico de las micro y pequeñas empresas latinoamericanas, a partir del análisis sistémico. Tomo I (pp. 23-33). Fontamara.

Rivas, A. (2019). La innovación en el mundo y Ecuador: análisis a partir del global innovation index. Escuela de Negocios ESPAE. https://bit.ly/3CgDHgg

Ron Amores, R., \& Sacoto Castillo, V. (2017). Las pymes ecuatorianas: Su impacto en el empleo como contribución del PIB pymes al PIB total. Revista Espacios, 38(53), 15.

Sistema de Información Local (SIL). (2020). Sistema de Información Local del Gobierno Descentralizado Autónomo Provincial de Santo Domingo. GAD Provincial de Santo Domingo. https://bit.ly/3G69Fyi

Smith, N.R., \& Miner, J.B. (1983). Type of entrepreneur, type of firm, and managerial motivation: Implications for organizational life cycle theory. Strategic management journal, 4(4), 325-340. 